\title{
Fascitis necrosante bilateral de diseminación hematógena por Staphylococcus Aureus. Caso pediátrico
}

\section{Necrotizing bilateral fasciitis of hematogenous dissemination, caused by Staphylococcus Aureus. Pediatric case}

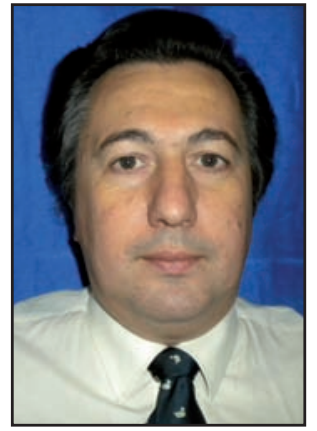

Manzani Baldi, J.R.

Manzani Baldi, J.R.*, Rodríguez, G. ${ }^{* *}$

Resumen

Presentamos un caso clínico muy poco frecuente de fascitis con miositis y necrosis cutánea en tronco y muslos, de presentación bilateral y simétrica y de mecanismo hematógeno, en una niña de 4 años durante el desarrollo de un cuadro séptico de foco primario orofaringeo, que requirió internamiento en centro de terapia intensiva pediátrica (CTP).

Optamos por una conducta quirúrgica agresiva y precoz, apoyada por medidas médicas de soporte vital y antibioticoterapia empírica hasta el momento del diagnóstico.

El agente etiológico fue Staphylococcus aureus. El resultado tras varias intervenciones, fue a costa de secuelas estéticas importantes.

El seguimiento de la paciente tras 6 años de evolución, no ha mostrado ningún otro episodio similar.

\author{
Palabras clave Fascitis necrosante, Staphylococcus \\ Aureus, Pediatria. \\ Código numérico 161
}

We present a not very frequent clinical case of fasciitis with miositis and cutaneous necrosis in trunk and thighs, with bilateral and symmetrical presentation and haematogenous dissemination mechanism in a 4 years old girl during the development of sepsis with primary oropharingeal focus, that required to be interned in paediatric intensive care unit.

It was necessary and aggressive an precocious surgical treatment, supported by vital life measures and empiric antibiotics, until the final germen diagnosis. The etiologic agent was Staphylococcus aureus.

As a result, there were very important aesthetic sequels after several surgical procedures.

After 6 years evolution, there wasn't any other similar episode.

\begin{tabular}{ll}
\hline Key words & $\begin{array}{l}\text { Necrotizing fasciitis, Staphylococcus } \\
\text { Aureus, Children. }\end{array}$ \\
Numeral Code & 161
\end{tabular}




\section{Introducción}

La fascitis necrosante en niños es, afortunadamente, un cuadro poco frecuente; pero cuando se instala, casi independientemente de su etiología, resulta un cuadro clínico grave que registra una elevada morbimortalidad (mortalidad aproximada del $21 \%$ en niños, al $25 \%$ en adultos) (1),

El pronóstico de este cuadro depende fundamentalmente de un diagnóstico clínico-paraclínico precoz (principalmente clínico), de ahí la importancia de no subestimar el conjunto de signos y síntomas que acompañan las primeras alteraciones de piel. Es aquí donde la experiencia del cirujano tratante puede ser un factor determinante, dado que la tasa de supervivencia cae exponencialmente después de 24 horas desde el inicio del cuadro cutáneo (6\% frente al $25 \%$ de mortalidad global) (1).

El diagnostico del caso que presentamos, con muy poca literatura al respecto, se hizo más difícil aún debido a la ausencia de antecedentes de lesiones previas, a modo de puerta de entrada, tales como: punciones, cirugía en el área, heridas, traumatismos, hematomas, picadura de insectos, contacto con agentes químicos o físicos, radiación ionizante o calórica o muy frecuentemente, lesiones cutáneas de varicela $(2,3)$.

En un estudio retrospectivo de 10 años realizado en el H.P.Rossell, en un 24,2\% de los casos no se determinó la puerta de entrada, pero en todos estos casos, las lesiones fueron unilaterales. Además, como plantearemos más adelante, la etiopatogenia de origen sistémico produce una necrosis que va de profundidad a superficie, es decir, los trastornos cutáneos aparecen tras una extensa miofascitis subyacente, como una simple escara húmeda flictenular de pocos centímetros de diámetro.

\section{Caso clínico}

Paciente de sexo femenino de 4 años de edad, procedente de la ciudad de Salto (Uruguay). Buen desarrollo pondo-estatural, con antecedentes personales de síndrome de aspiración de líquido meconial, crecimiento y desarrollo normales, bien inmunizada y alimentada.

Comienza 4 días antes del ingreso hospitalario con angina pultácea, febril, por lo que recibe tratamiento con claritromicina vía oral.

El día antes del ingreso hospitalario aparecen además lesiones equimóticas dolorosas en la cara externa del muslo derecho y en la cara interna de muslo izquierdo, con lesiones petequiales a ese nivel.

Ingresa en el Hospital de la ciudad de Salto donde se constatan las lesiones hemorragíparas descritas y una lesión úlceronecrotica de amígdala izquierda, rigidez de nuca y apirexia.

Se plantea como probable diagnóstico meningococcemia y se inicia antibioticoterapia con penicilina, ceftriaxona y gentamicina vía intravenosa. Se realiza también punción lumbar ( PL), cuyo resultado fue nor- mal, AELO 400 unidades, crasis: hipocoagubilidad con consumo de factores, leucocitosis 15000 , sodio plasmático125meq, potasio 4,5 meq.

Se reponen glóbulos rojos concentrados, plasma, crioprecipitados y plaquetas, a pesar de lo cual mantiene los trastornos de la crasis y presenta sufusiones hemorrágicas con flictenas en ambas regiones lumbares y en muslos, por lo que ingresa en el centro de terapia intensiva (CTI), lúcida, en extrema gravedad, con polipnea superficial, saturación del 90\%, palidez cutáneo-mucosa. Las sufusiones hemorrágicas se consolidan, con flictenas abiertas y elementos de necrosis cutánea, sobre todo en la cara externa de ambos muslos y en regiones dorso-lumbares laterales, acompañadas de intenso dolor en dicha zona.

Comenzamos con reposición hematológica enérgica, hidratación parenteral y luego alimentación parenteral y se mantiene la antibioticoterapia hasta obtener los resultados de los estudios bacteriológicos; apoyo inotrópico, oxigenación con catéter nasal, protección gástrica e inmunoglobulinas vía intravenosa.

Es en esta etapa de evolución cuando se opera a la paciente, realizando resección de extensas áreas necróticas (Fig. 1), que exceden en profundidad lo que se extiende en superficie; de esta manera resecamos parcialmente tejidos musculares necróticos de glúteos mayores, fascia lata, oblicuo mayor y dorsal ancho (Fig. 2), donde encontramos fascia de color grisáceo con áreas de necrosis fácilmente disecables (4) y realizamos intenso lavado quirúrgico con clorhexidina y suero fisiológico.

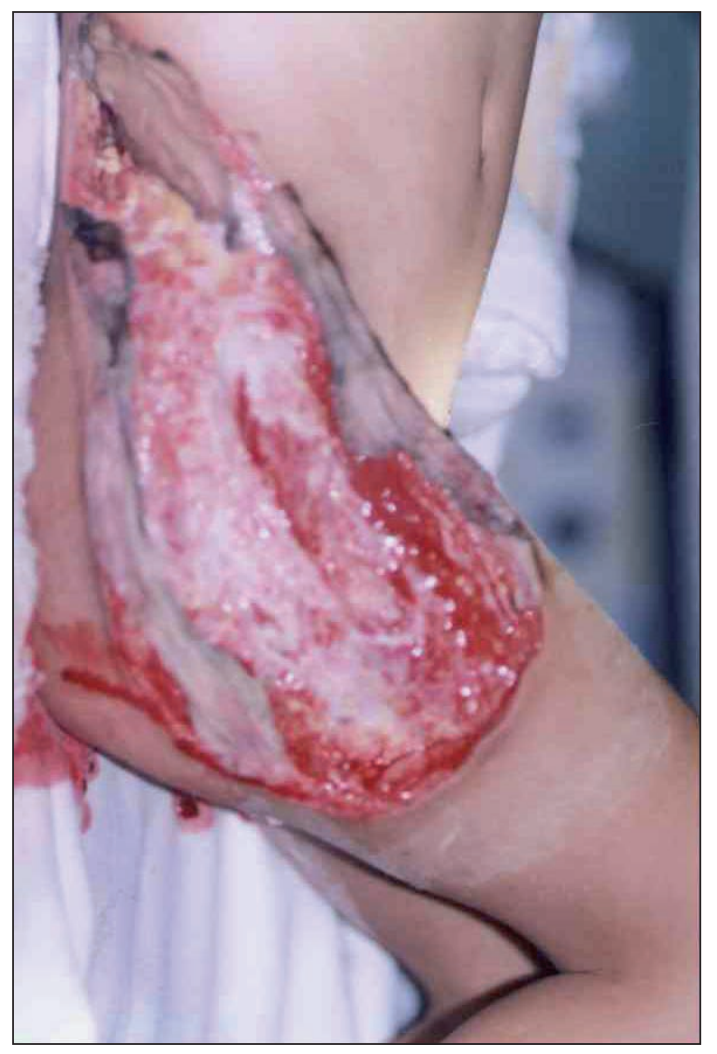

Fig. 1. Fascitis necrosante en niñá de 4 años. Aspecto de la zona afectada tras la resección quirúrgica practicada en el lado derecho. 

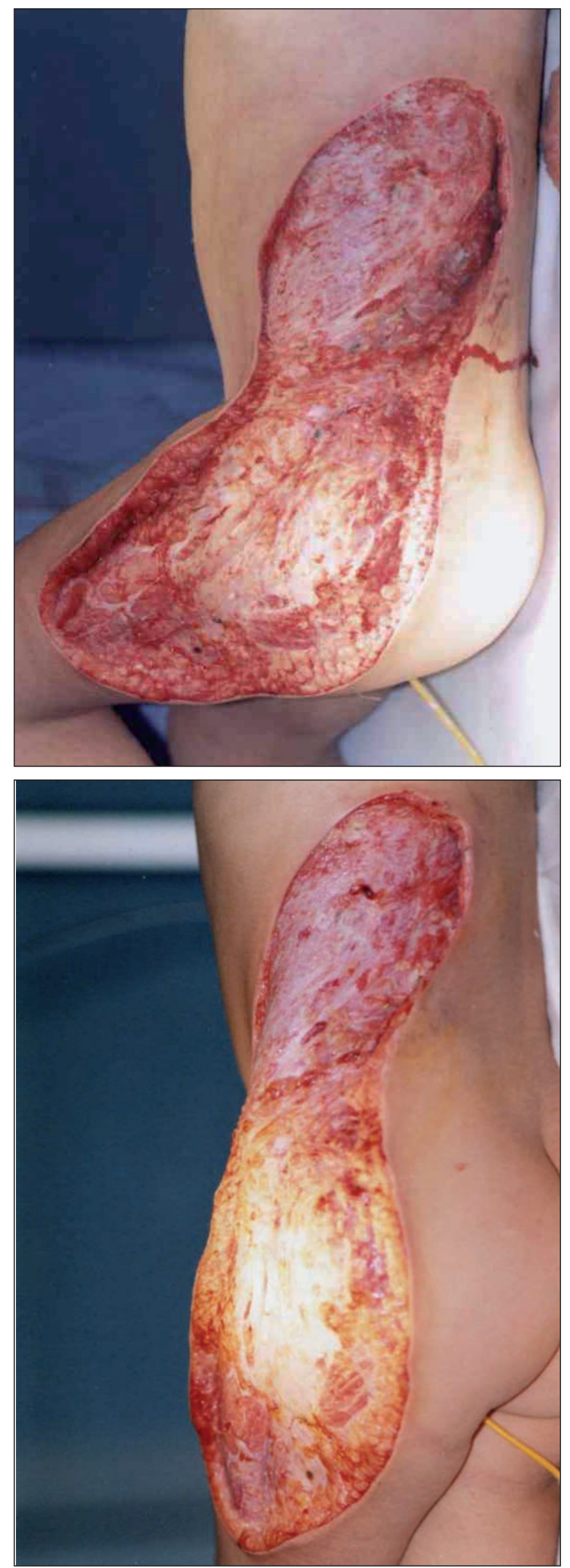

Fig. 2. A) Aspecto de la resección practicada en el lado izquierdo.

B) Dimensiones de la pérdida de sustancia con la pierna extendida.

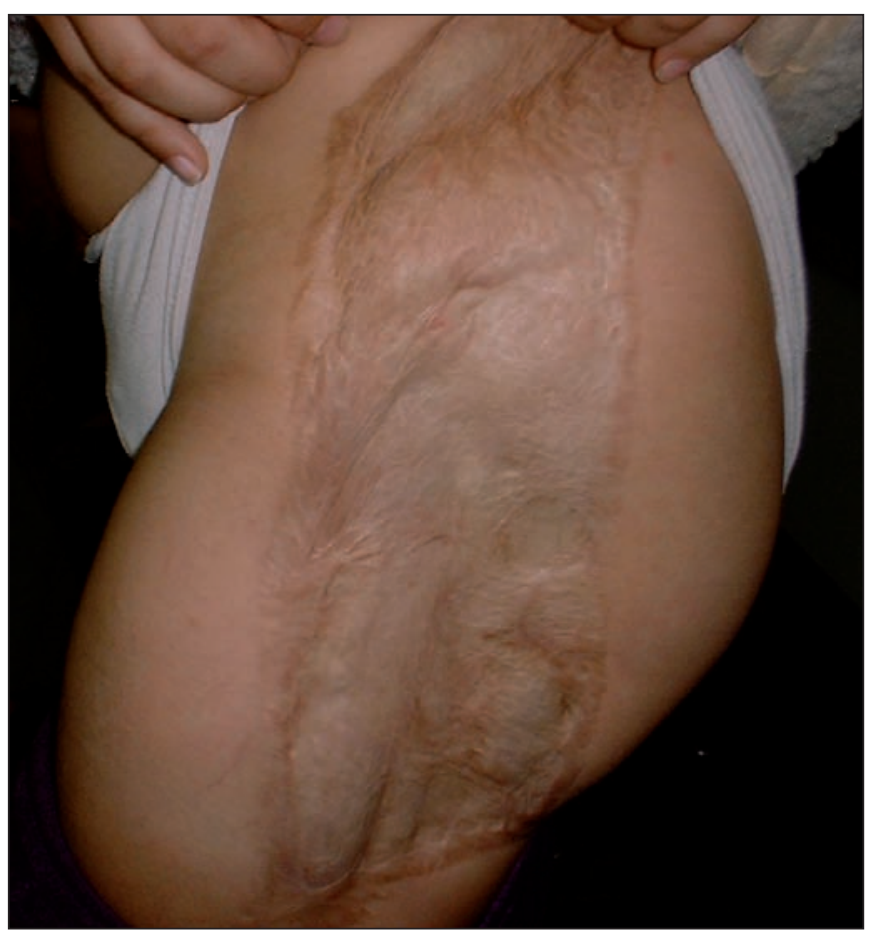

Fig. 3. Resultado a largo plazo: 6 años de postoperatorio.

Este procedimiento se repite en 3 oportunidades, cada 48 horas, hasta dejar una zona libre de tejido necrótico, tanto en profundidad como en superficie.

La paciente comienza a mejorar clínicamente y los exámenes paraclínicos muestran el resultado de un hemocultivo que desarrolla Staphylococcus aureus, por lo que se rota la gentamicina por vancomicina. Los cultivos del foco fueron negativos. (5)

Posteriormente, injertamos las zonas de pérdida de sustancia con piel de donante, realizándose a los 7 días la remoción de los homoinjertos y su sustitución por autoinjertos expandidos 2:1 tomados de cara anterior de los muslos.

Se otorga el alta definitiva a la paciente a los 15 días del ingreso.

Tras 6 años de seguimiento, no ha presentado más episodios de infección grave en las zonas afectadas ni en el resto de su economía corporal (Fig. 3).

\section{Discusión}

Comúnmente se acepta que la liberación de endo y exotoxinas bacterianas es dependiente de un foco infeccioso grave de partes blandas y son éstas las responsables de del fallo orgánico multisitémico.

En el caso clínico que describimos, el mecanismo se desarrolla de manera inversa, partiendo de una sepsis generalizada, el concurso de toxinas-gérmenes coloniza un área de partes blandas en forma bilateral y simétrica.

Esta inusual forma de presentación en el curso de una sepsis y sin puerta de entrada local, junto con la característica de bilateralidad, nos lleva a plantear una hipóte- 
sis fisiopatológica distinta a las causas habituales de fascitis necrosante.

Sabemos que el germen aislado en el hemocultivo, Staphylococcus aureus, produce una serie de toxinas responsables de los fenómenos dermatológicos y musculares.

Partiendo de la base de una paciente que cursa una sepsis estafilocóccica con un cuadro de disfunción orgánica múltiple por las toxinas, vinculamos a éstas los procesos de celulitis y necrosis.

La acción de la TSCT1(toxina síndrome choque tóxico), que induce la producción de interleuquina 1 por los monocitos, que a su vez influye en el metabolismo del ácido araquidónico celular, es responsable de la proteolisis muscular. A su vez, la exfoliatina, toxina responsable de las manifestaciones cutáneas, que actúa separando la porción intraepidérmica a nivel del estrato granuloso, facilita la formación de flictenas y el desollamiento, cuya manifestación clínica benigna se conoce como síndrome de la piel escaldada (6).

Las toxinas A y B son epidermolíticas, rompen los desmosomas de las células de la epidermis, lo que provoca una necrolisis masiva parecida a la que se produce en las quemaduras de segundo grado.

A nivel microvascular se produce una vasculitis con infiltración y necrosis de las paredes vasculares, que se acompaña de endotelitis y trombosis. Todo esto origina isquemia localizada y hemorragia, que junto a la acción de las toxinas, desencadena un proceso de coagulación y hemorragias diseminadas (CID) y a nivel local determina las lesiones cutáneas ampulares, nodulares y la epiescleritis, debido al depósito de complejos inmunes.

Todo este mecanismo lleva, paulatinamente, a una disfunción en el intercambio gaseoso y por ende, a la necrobiosis músculo-cutánea.

En el caso que presentamos, es la acción conjunta de ambas Toxinas que, por motivos que desconocemos, eligen como objetivo la regiones laterales de tronco y los muslos (curiosamente de forma similar a lo que se ve en la meningococcemia, en el síndrome de Waterhouse Friderichsen), desarrollándose un proceso que lleva al cuadro descrito. Este fenómeno, tanto a nivel local como sistémico, genera un ciclo que, si no es interrumpido, lleva a la muerte.

La acción, de profundidad a superficie, explica que durante la exploración quirúrgica constatemos que el área de necrosis muscular excedía el área cutánea afectada.Lo que nosotros llamamos comúnmente celulitis necrosante, podría catalogarse más precisamente, ta y como lo denominan autores anglosajones, un NSTI (Necrotizing Soft Tissues Infection) (7); es decir, el proceso de necrosis comienza en la profundidad de los tejidos blandos, fascia y músculo y de allí se extiende a la superficie.

Coincidimos con Barie (7), en que el score LRINEC (Laboratory Risk Indicator for Necrotizing Fasciitis) (8), basado en valores paraclínicos como el aumento de glóbulos blancos mayor de 14000, hiponatremia menor a
135 meq. y elevación de la creatininemia) para advertir la gravedad y la progresión mórbida y letal de estos cuadros, tiene un valor pronóstico relativo y en el mejor de los casos, sirve como un elemento más a tener en cuenta. En nuestra paciente tenemos 2 de los 3 datos paraclínicos positivos: la leucocitosis de 15000 y la hiponatremia)

Por todos estos mecanismos descritos, no es de extrañar que en este caso no aparezcan gérmenes en el cultivo de los tejidos resecados como ocurre en las celulitis con puerta de entrada cutánea, donde en un $70 \%$ de las ocasiones los cultivos son positivos (9).

La manera de cortar el círculo a nivel local es la eliminación de todos los tejidos afectados que inducen al depósito de complejos inmunes y perpetúan la acción de las toxinas a nivel del tejido sano concomitante (10).

Independientemente del mecanismo o de la etiología que lleva a este tipo de cuadro clínico, el concepto quirúrgico es siempre el mismo: debe ser agresivo y precoz, con debridamientos amplios y resección de tejidos necróticos hasta llegar a un plano de tejido sano; todo ello acompañado por una intensa y bien dirigida antibioticoterapia junto a medidas de apoyo vital $(11,12)$.

Ante la sospecha de fascitis y miositis, se debe profundizar la resección hasta un margen seguro de viabilidad de los tejidos.

\section{Conclusiones}

Queremos destacar lo raro de la etiopatogenia del cuadro clínico que presentamos, sobre el que disponemos de muy poca literatura, así como enfatizar el concepto de la conducta a seguir ante las sospecha de este tipo de casos, que debe ser enérgica e inmediata(13), no demorada por estudios paraclínicos, dado que la mortalidad en los casos de fascitis necrotizante es exponencial al tiempo de evolución. Tampoco se tendrán en cuenta en estos cuadros elementos estéticos o funcionales, puesto que el objetivo primario es la conservación de la vida.

\section{Agradecimientos}

Nuestro agradecimiento al Prof. Dr. Juan J. Hornblas, quien nos apoyó en el tratamiento de esta paciente al solicitar su presencia desde el inicio del cuadro y a los Prof. Carlos Carriquiry y Mario Londinsky que leyeron y corrigieron este trabajo.

\section{Dirección del autor}

Dr. Jesús Ricardo Manzani Baldi

C/ Moltke 1233, Montevideo, Uruguay.

Código Postal 12401

e-mail: jmanzani@adinet.com.uy 
Bibliografía

1. Michelena, C., Asti, H.: "Celulitis graves en la edad pediátrica". Artículo original."Archivos de Pediatría Uruguaya, 2001; 72(s): S34

2. Prego, J; Sehabiague, G,; Leonardis, D; Gutierrez, C: "Varicela complicada con fascitis necrotizante. Importancia de un diagnóstico oportuno". Archivos de Pediatría Uruguaya 2001; 72(s):S84.

3. Goncalves E, Furtado F, Estrada J, Vale MC, Pinto M, Santos M, Moura G, Vasconcelos C.: "Necrotizing fasciitis after varicella". Acta Med Port. 2001;14(3):367.

4. Ceccini, E., Gonzalez, Silvia: ' 'Infectología y enfermedades infecciosas" infecciones de partes blandas. Edic. journal 2008 p 271.

5. Kiriakopolous, E.; Brown, M.H.; Starr, J.A.;Choptiany, P.J.; Mederski,B: "Spontaneous bilateral necrotizing fasciitis of the forearm: a case report."Can J Plastic Surg 1997;5(4):246.

6. Farreras Valenti, P.: "Medicina Interna".Editorial Marín SA, 1985.Vol. II, Pp.809-814.
7. Barie, Philip S.: "The Laboratory Risk Indicator for Necrotizing Fascitis (LRINEC) score: Useful tool or paralysis by analysis?" Critical Care Medicine, 2004 32(7):1618.

8. Anaya, D,.A.; McMahon, K.; Nathens, A.B; Sullivan, S.R. ; Foy, H.; Bulger, E.: " Predictors of Mortality and Limb Loss in Necrotizing Soft Tissue Infections", Archives of Surgery 2005, 40(2): 151-157.

9. Torterolo, E; Morelli, R; Silva, C: "Infecciones graves de partes blandas".Cir Uruguay 1977;47(3):183

10. Morelli, R.; Torterolo, E; Silva,C: "Celulitis necrotizante".Cir. Uruguay 1977;47(3):197.

11. Romero Torres, R.: "Tratado de Cirugía", vol II, Interamericana, 1987.Cap. II Pp. 29-56.

12. Harrison, T. R.: "Principios de Medicina Interna". Vol.I.Interamericana-Mc. Graw- Hill ,1989.Pp.663-678.

13. Rodríguez Lorenzo, A., Midón Míguez, J. and Martelo Villar, F.: "Síndrome del shock tóxico estreptocócico tras fascitis necrotizante por estreptococo pyogenes". Cir. plást. iberolatinoam., 2007, 33 (4):257. 